\title{
InGaAsP/InP Membrane Gain Sections for III-V/SiN Heterogeneous Photonic Integration
}

\author{
Christopher Heidelberger ${ }^{1}$, Christos T. Santis ${ }^{2}$, Jason J. Plant ${ }^{1}$, Erin M. Morissette ${ }^{1}$, \\ Dave Kharas $^{1}$, Reuel B. Swint ${ }^{1}$, Amnon Yariv ${ }^{2}$, Paul W. Juodawlkis ${ }^{1}$ \\ ${ }^{1}$ Lincoln Laboratory, Massachusetts Institute of Technology, Lexington, Massachusetts 02420, USA \\ ${ }^{2}$ Department of Applied Physics and Materials Science, California Institute of Technology, Pasadena, California, 91125, USA \\ Author e-mail address: c.heidelberger@ll.mit.edu
}

\begin{abstract}
We present a fabrication process for $200 \mathrm{~nm}$ thick InGaAsP/InP membrane gain sections $(\lambda=1550 \mathrm{~nm})$ suitable for heterogeneous integration with $\operatorname{SiN}_{\mathrm{x}}$ PICs. The structures exhibit desired electrical performance and support lasing. (C) 2021 The Author(s)
\end{abstract}

\section{Introduction}

Photonic integrated circuits (PICs) using silicon nitride $\left(\mathrm{SiN}_{\mathrm{x}}\right)$ or other dielectric waveguides offer benefits over more conventional silicon ( $\mathrm{Si}$ ) waveguides, including lower loss, better power handling, and broader wavelength transparency [1]. Heterogeneous integration (i.e., surface bonding) of III-V semiconductors with Si PICs allows for parallel fabrication of multiple gain sections [2]. However, this approach has not been successfully demonstrated with $\mathrm{SiN}_{\mathrm{x}}$ PICs due to the low refractive index of $\mathrm{SiN}_{\mathrm{x}}$ relative to the III-V materials, which makes it difficult to couple light evanescently from III-V into $\mathrm{SiN}_{\mathrm{x}}$. Thin III-V membrane lasers with lateral current injection have been demonstrated on Si substrates [3,4]. With such a structure, it is possible to engineer an adiabatic transition from a III$\mathrm{V}$ fundamental mode into a $\mathrm{SiN}_{\mathrm{x}}$ waveguide using a III-V taper with modest tip width. In this work, we develop a fabrication process for an InGaAsP/InP electrically-pumped gain section (1550 nm) suitable for integration with $\mathrm{SiN}_{\mathrm{x}}$ PICs (Figure 1a), demonstrating progress towards fabrication of hybrid III-V/SiN $\mathrm{S}_{\mathrm{x}}$ lasers and other integrated systems.

\section{Device Fabrication}

The InGaAsP/InP multi-quantum well (MQW) structure was grown on InP substrates by metalorganic chemical vapor deposition (MOCVD) with the following layers: a $50 \mathrm{~nm}$ sacrificial InGaAs etch stop, a $50 \mathrm{~nm}$ InP bottom clad, a $100 \mathrm{~nm}$ InGaAsP MQW region with five 0.4\% compressively strained wells having peak photoluminescence at $1550 \mathrm{~nm}$, and a $50 \mathrm{~nm}$ InP top clad. For process development, surrogate $\operatorname{SiN}_{\mathrm{x}}$ PICs (with no SiN $\mathrm{x}_{\mathrm{x}}$ layer) were prepared by plasma-enhanced chemical vapor deposition (PECVD) deposition of $\mathrm{SiO}_{2}$ on a $\mathrm{Si}$ substrate followed by chemicalmechanical polishing (CMP) to a final $\mathrm{SiO}_{2}$ thickness of $3 \mu \mathrm{m}$ with an RMS roughness of $400 \mathrm{pm}$. The III-V wafer was then bonded to the surrogate PIC. $\mathrm{O}_{2}$ plasma is frequently used to prepare surfaces prior to covalent oxide-oxide bonding [5]. However, in this case $\mathrm{O}_{2}$ plasma processing was found to cause significant degradation of carrier lifetime in the InGaAsP MQW (Figure 1b) due to the close proximity of the MQW layers to the III-V surface. UV-ozone pretreatment was chosen because of its reduced impact on carrier lifetime and sufficient strength of the resulting oxideoxide bond. Figure 1c shows an image of the sample after chemical substrate and etch stop removal, showing the high bond yield (>95\%) and low defect density $\left(\sim 75 \mathrm{~cm}^{-2}\right)$. Next, the InP top clad and InGaAsP MQW layers were patterned into stripes using a $400 \mathrm{~nm}$ thick $\mathrm{SiN}_{\mathrm{x}}$ hardmask, leaving behind the InP bottom clad. $150 \mathrm{~nm}$ of undoped InP was grown selectively by MOCVD on either side of the stripe. Cross-section SEM shows complete filling of the undercut regions with regrown InP (Figure 1d and e). Both $<011>$ stripe orientations were investigated, with the [011] direction being preferred due to nearly vertical InGaAsP MQW sidewalls. $\mathrm{Si}+$ and $\mathrm{Be}+$ ion implants were used to define the $\mathrm{n}$ -

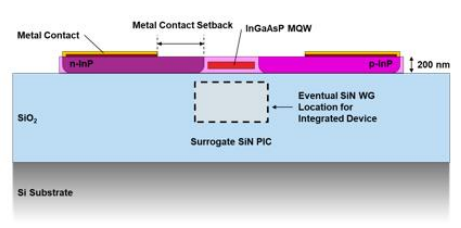

b.

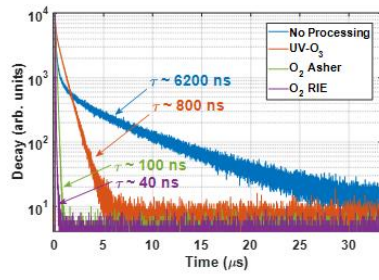

c.

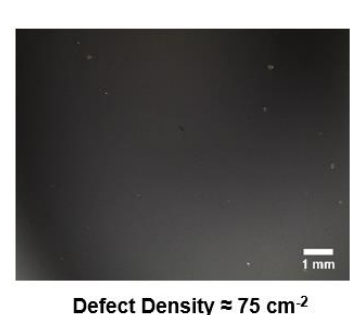

d.

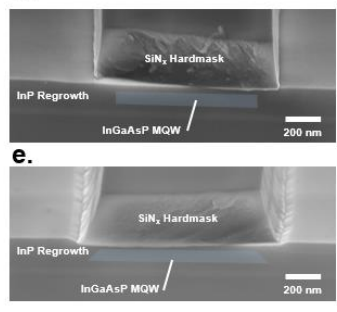

Figure 1: a) Lateral cross-section schematic of InGaAsP/InP membrane gain section showing location of SiN $\mathrm{N}_{\mathrm{x}}$ waveguide for heterogeneous integration. Note that the $\mathrm{SiN}_{\mathrm{x}}$ waveguide is not present in this work. b) TRPL decay of InGaAsP/InP MQW structure before and after various pre-bond surface treatments. c) Image of bonded III-V membrane after InP substrate removal showing large bonded area and low defect density. d) Cross-section SEM of patterned InGaAsP MQW (highlighted) surrounded by InP regrowth with ridge in $\lceil 011\rceil$ direction. e) Same as (d) but with ridge in $\lceil 0-11\rceil$ direction. 
and p-type lateral contact regions, respectively. For both implants, the $\mathrm{SiN}_{\mathrm{x}}$ hardmask acted as a self-aligned implant barrier over the MQW stripe resulting in a doping setback of $\sim 100 \mathrm{~nm}$ from the MQW region (see Figure 1d). Ohmic contacts were then deposited. A standard $\mathrm{Ni} / \mathrm{Ge} / \mathrm{Au}$ metallurgy was used for the $\mathrm{n}$-type contacts while a $\mathrm{Au} / \mathrm{Zn} / \mathrm{Au}$ metallurgy was used for the p-type contacts. Transmission line measurements (TLM) showed sheet resistances of $3380 \Omega / \mathrm{sq}$ and $180 \Omega / \mathrm{sq}$ and contact resistivities of $7 \times 10^{-6} \Omega \mathrm{cm}^{2}$ and $5 \times 10^{-5} \Omega \mathrm{cm}^{2}$ for $\mathrm{n}$ - and p-type InP contacts, respectively. Finally, the InP membrane was removed from the area surrounding the devices. Most devices included InP tapers at each end of the InGaAsP MQW stripe, which will enable an adiabatic transition into an underlying $\mathrm{SiN}_{\mathrm{x}}$ waveguide in a future iteration. Others included abrupt etched InP facets to form Fabry-Perot lasers in the III-V for testing purposes.

\section{Device Testing}

$\mathrm{I}-\mathrm{V}$ data from a $5 \mathrm{~mm}$ long $\mathrm{InGaAsP} / \mathrm{InP}$ membrane gain section with tapered ends and a $2 \mu \mathrm{m}$ metal setback is shown in Figure 2a. The I-V behavior closely follows simulated data of the nominal structure (self-consistent solution of Poisson, drift, and diffusion equations) indicating that the dopant concentrations and setback distances from the MQW stripe are as-targeted. Series resistance scaled as expected with varying metal setback distances $(1.2 \Omega$ for $1 \mu \mathrm{m}$ setback to $3.3 \Omega$ for $3 \mu \mathrm{m}$ setback). Top-down images of the spontaneous emission show uniform power along the length of the device indicating uniform electrical injection and recombination mechanics. Figure $2 b$ shows one such image and Figure $2 \mathrm{c}$ shows the relative power as a function of position along the InGaAsP MQW stripe. Devices having abrupt rather than tapered ends exhibited lasing behavior (Figure 2d) with a threshold current density per quantum well of $\sim 380 \mathrm{~A} / \mathrm{cm}^{2}$. This high value is not unexpected given the relatively low $0.4 \%$ compressive strain in the quantum wells [6] and low reflectivity of the etched facets, and is comparable to that demonstrated in similar membrane lasers [3].

a.

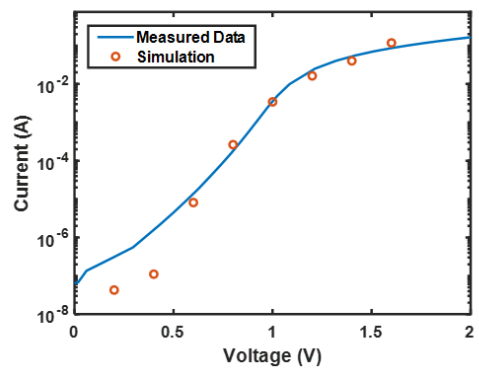

b.

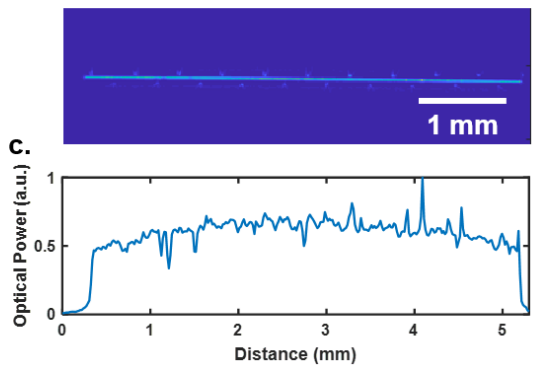

d.

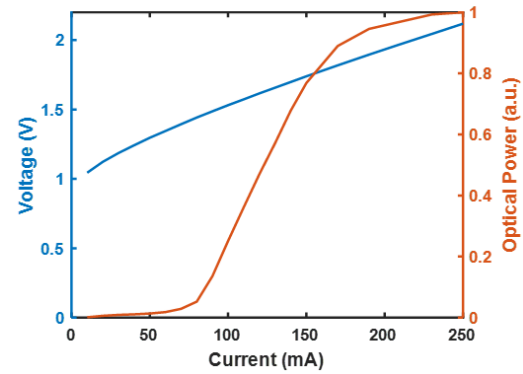

Figure 2: a) I-V curve for a $5 \mathrm{~mm}$ long device compared to simulation of nominal structure assuming $5000 \mathrm{~cm} / \mathrm{s}$ InP surface recombination velocity. b) Top-down IR image of spontaneous emission from a $5 \mathrm{~mm}$ long device under $100 \mathrm{~mA}$ drive current. $\mathrm{V}$-shaped spots along top and bottom of stripe are caused by light scattering from electrical probe tips. c) Relative optical power along length of device from (b). d) L-I-V data from $5 \mathrm{~mm}$ long InGaAsP/InP membrane laser.

\section{Conclusion}

We have developed a process for fabrication of $\mathrm{InGaAsP} / \mathrm{InP}$ membrane gain sections on $\mathrm{SiO}_{2} / \mathrm{Si}$ substrates compatible with heterogeneous integration with $\mathrm{SiN}_{\mathrm{x}}$ PICs. The resulting devices exhibit good electrical performance, consistent with simulation, indicating good material quality and correct geometry. Devices without end mirrors showed uniform spontaneous emission along their length and devices with end mirrors exhibited room-temperature CW lasing.

DISTRIBUTION STATEMENT A. Approved for public release. Distribution is unlimited. This material is based upon work supported by the Defense Advanced Research Projects Agency (DARPA) under Air Force Contract No. FA8702-15-D-0001. Any opinions, findings, conclusions or recommendations expressed in this material are those of the author(s) and do not necessarily reflect the views of DARPA.

Delivered to the U.S. Government with Unlimited Rights, as defined in DFARS Part 252.227-7013 or 7014 (Feb 2014). Notwithstanding any copyright notice, U.S. Government rights in this work are defined by DFARS 252.227-7013 or DFARS 252.227-7014 as detailed above. Use of this work other than as specifically authorized by the U.S. Government may violate any copyrights that exist in this work.

\section{References}

[1] C. Sorace-Agaskar et al., "Versatile Silicon Nitride and Alumina Integrated Photonic Platforms for the Ultraviolet to Short-Wave Infrared," IEEE J Quantum Elect, vol. 25, no. 5, pp. 1-15 (2019)

[2] T. Komljenovic, D. Huang, P. Pintus, M. A. Tran, M. L. Davenport, and J. E. Bowers, "Photonic Integrated Circuits Using Heterogeneous Integration on Silicon," Proc IEEE, vol. 106, no. 12, pp. 2246-2257 (2018)

[3] S. Matsuo, T. Fujii, K. Hasebe, K. Takeda, T. Sato, and T. Kakitsuka, "Directly modulated buried heterostructure DFB laser on $\mathrm{SiO}_{2} / \mathrm{Si}$ substrate fabricated by regrowth of InP using bonded active layer," Opt Express, vol. 22, no. 10, p. 12139 (2014)

[4] T. Fujii et al., "Evaluation of Device Parameters for Membrane Lasers on Si Fabricated with Active-Layer Bonding Followed by Epitaxial Growth," IEICE T Electron, vol. E100.C, no. 2, pp. 196-203 (2017)

[5] M. Yokoyama et al., "Formation of III-V-on-insulator structures on Si by direct wafer bonding," Semiconductor Sci Tech, vol. 28, no. 9, p. 094009 (2013)

[6] A. R. Adams, "Strained-Layer Quantum-Well Lasers," IEEE J Quantum Elect, vol. 17, no. 5, pp. 1364-1373 (2011) 